\title{
Carrier-separating demodulation of phase shifting self-mixing interferometry
}

\author{
Yufeng Tao Ming Wang* Wei Xia \\ Jiangsu Key Laboratory on Opto-electronic Technology, Department of Physics Science, Nanjing, 210023 \\ *Corresponding author: Mangming@njnu.edu.cn
}

\section{Abstract:}

A carrier separating method associated with noise-elimination had been introduced into a sinusoidal phase-shifting selfmixing interferometer. The conventional sinusoidal phase shifting self-mixing interferometry was developed into a more competitive instrument with high computing efficiency and nanometer accuracy of $N 100$ in dynamical vibration measurement. The high slew rate electro-optic modulator induced a sinusoidal phase carrier with ultralow insertion loss in this paper. In order to extract phase-shift quickly and precisely, this paper employed the carrier-separating to directly generate quadrature signals without complicated frequency domain transforms. Moreover, most noises were evaluated and suppressed by a noiseelimination technology synthesizing empirical mode decomposition with wavelet transform. The overall laser system was described and inherent advantages such as high computational efficiency and decreased nonlinear errors of the established system were demonstrated. The experiment implemented on a high precision PZT (positioning accuracy was better than 1nm) and compared with laser Doppler velocity meter. The good agreement of two instruments shown that the short-term resolution had improved from $10 \mathrm{~nm}$ to $1.5 \mathrm{~nm}$ in dynamic vibration measurement with reduced time expense. This was useful in precision measurement to improve the SMI with same sampling rate. The proposed signal processing was performed in pure timedomain requiring no preprocessing electronic circuits.

Key words: carrier-separating; empirical mode decomposition; wavelet transform; phase extraction; computing efficiency.

\section{Introduction}

Based on precious theories on SMI (self-mixing interferometry) [1-3], threshold gain of laser was significantly affected by optical feedback from plane mirrors, metals or even rough surfaces. Half wavelength displacement in external optical length generated one self-mixing fringe. Meanwhile, strength of feedback light affected the shape of fringes which could be sinusoidal or saw-tooth. In the respect of application, compact SMI [4][5] required only a laser and a mirror but provided high sensitivity to phase[6] like conventional dual-beam interferometers. Due to the beneficial advantages such as inexpensive, being traceable to laser wavelength and ability of working with non-cooperative objects, SMI had appeared frequently in non-contact measuring physical quantities such as velocity [7], displacement [8], structure [9], micro damping vibration [10], biological signal [11], rotating angle, range finding [12], refraction index and even profile. In our literature review, increasing advanced methods had been applied into SMI for improvement containing the smoothing periodogram method [13], MUSIC frequency estimation method[14], the electronic closed-loop method [15], sinusoidal phase shifting method [16], Bessel-FFT analysis [17], error compensating algorithm [18]and electronic frequency down conversion [19] .

Specifically, 1nm resolution by phase shifted SMI [20] was reported under a stationary condition: speed of optical path length changing was extremely slow and unidirectional, therefore, dynamic movement with a higher vibrating frequency or amplitude would make resolution plummet unavoidably and signal processing based on five phase-shifted method [21] was not simplest. Another sub-nanometer resolution(better than $1 \mathrm{~nm}$ ) theoretically achieved by differential SMI structure [22] realized 
on precision displacement stage was experimentally limited in low-speed range $(<50 \mathrm{~Hz})$. In differential SMI, reference laser must operate at identical wavelength in same feedback regime with measuring laser to estimate time delay, besides, alignment of two optical path also technologically complicated the design of mechanics. A particularly fine resolution( $(0.2 \mathrm{~nm})$ of SMI using vertical-cavity surface-emitting laser to measure displacement of nano-mechanical cantilever[23] was demonstrated by L.David and his co-workers, where laser was placed closely to non-coated surface of cantilever(only 35um) to introduce strong feedback level SMI with inclining fringes. Since the displacement was read out from voltage of SMI intensity, if laser voltage visibility dropped with decreased feedback level in harsh environments or in remote distance, this resolution became questionable. Some other current injecting technologies [24] inducing undesired intensity modulation complicated laser system with re-shaping technique to compensate nonlinear noise and created difficulties in dynamical measurement. In practical applications, these SMI systems also suffered from the unwanted optics or mechanical noises.

However, many cutting-edge industries like the nanotechnology, micro-electro-mechanical manufacturing, biotechnology and precision engineering had great demand on high-resolution dynamical measurement even under poor reflection(feedback level is weak). In essence, dynamical measurement required two main key factors: high computing efficiency for real-time and a fine short-term resolution to reflect measured quantities precisely. Correspondingly, the first objective of this paper was decreasing computing time in time-domain by phase demodulation. We duplicated the phase-shifting SMI and measured the micron-vibration. Collected laser intensity fluctuation was processed by a simplified carrier separating method. Phase modulation depth was constant $0.5 \mathrm{rad}$ to extract quadrature signals with minimal calculation steps with absence of the complicated FFT, Z-transform or Bessel expansion functions. Due to algebraic computation, time expense was much reduced to support real-time performance. Generated quadrature phases permitted a precise phase measurement through use of arctangent function without complex algorithms, which significantly boosts the measuring efficiency.

Electronic driven voltage of phase modulation, unstable laser power and environmental disturbances could induce pseudo vibration to degenerate the performance. Therefore, the second objective of this paper was suppressing the nonlinear errors as effective as possible. By synthesizing EMD (empirical mode decomposition) with WT (wavelet transform) [25] to decompose noises out in extracted quadrature signals, useful self-mixing fringes were less changed compared to common filters. In process of decomposition, EMD was data-driven to decompose out signals automatically, while WT determined the decomposing levels by fringe counting. To examine effectiveness of EMDWT, laser Doppler velocity meter was introduced as referring results. Repetitive experimental deviations of two instruments proved short-term resolution was decreased from $10 \mathrm{~nm}$ to $1.5 \mathrm{~nm}$ in measurement of different shape micron vibrations.

This paper was organized following our two objectives of improvement. In experiment section, schematic of self-aligned setup and typical SMI signal was presented without cat-eye reflector or focusing lens. This section illustrated measurement of nanometer vibration by phase shifting SMI as beginner. Then, mathematical model of SMI signal was deducted and processed by carrier-separating, which featured with pure algebraic operations and $0.5 \mathrm{rad}$ phase modulation depth. Moreover, influence of various noise sources was summarized and the operating principle of EMDWT was introduced to improve signal-to-noise ratio. At last, experimental results from SMI and Ploytec-5000 laser Doppler velocity meter were compared as further verification.

\section{Experiment}

The whole optical path of phase shifting self-mixing system (seen in Fig.1) is placed on an air-floating platform to isolate non-axis vibrations as Refs. [17-19]. Laser source lasing at 632.8nm (TEM00 mode) is fed by $4.5 \mathrm{~mA}$ current to emit visible red light. VOA (variable optical attenuator) denotes a neutral density filter and placed next to front facet of laser, which is to deduce

optical strength ensuring that SMI operates in weak feedback regime. High coherent laser beam propagates through aperture (1mm-diameter)of the sealed electro-optic crystal (4002nf, Newfocus) and impinges on a diffusive mirror (which can be metal surface, glass or other untreated targets). Reflected light passes through the crystal once again and re-enters into laser cavity to generate self-mixing fringes. Vibration of the closed-loop served PZT induces a phase shift to laser intensity fluctuation. From 
Fig.1 we can see reflected light shares same optical axis with emitted light making SMI easily-alignment and only difference of this experimental optical path to the conventional one is lack of light-enhancing device like focusing lens or cat-eye reflector.

The used modulator has an adjustable slew rate up to $0.25 \mathrm{GHz} / \mathrm{s}$ depending on the applied voltages. Different to existing frequency modulation by current injection, phase modulation induces a pure phase carrier without intensity modulation. Based on electro-optic effect, refraction index of inserted MgO:LiNbO3(magnesium-oxide-doped lithium Niobate) crystal in external cavity varies according to the applied electric field following this relationship:

$$
\Delta n=\left(\frac{1}{2} n_{e}{ }^{3} r_{33}\right) E
$$

$r_{33}$ is a polarization coefficient of crystal material, $n_{e}$ is initial refraction index without applied electronic filed, $\Delta n$ is the variation of $n_{e}$ and linearly proportional to the strength of outside electrical field $E$. Although physical length of external cavity is permanent, varying refraction index of crystal changes the equivalent external optical length periodically. Therefore, a phasecarrier is induced and determined by two dimensional sizes of the crystal: the length and thickness as below:

$$
\Delta \varphi=\frac{2 \pi}{\lambda}\left(\frac{1}{2} n_{e}^{3} r_{33}\right) \frac{d}{h} V
$$

h denotes the thickness of the electro-optic crystal. As the Eq.2 shows, the shape, frequency and depth of the phase-carrier depends on the applied voltage $v$. The applied sinusoidal voltage changes the refraction index of the crystal and induces a highfrequency sinusoidal carrier into SMI. The phase shifting laser intensity from end facet of He-Ne laser impinges on a Si-based $\mathrm{GHz}$ broadband detector to generate photo current based on photoelectric effect. An adjustable impedance (VT, Thorlabs) implements the I/V conversion and send the voltage to DAQ device (USB-6361) for analog-digital conversion. Since the phase shift induced by vibration is much slower than phase-carrier in frequency domain, typical phase-shifting SMI signal features with the high-density optical carrier as illustrated in Fig.2 where no fringe order messing up is observed by finely controlling feedback level.

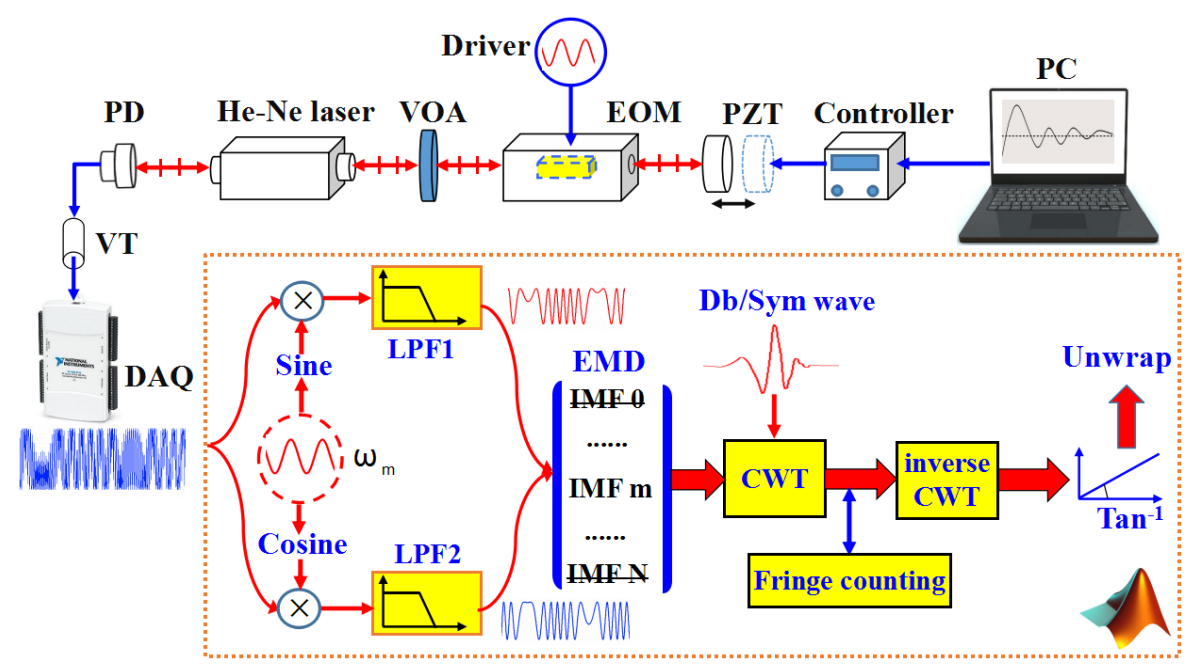

Fig. 1. Basic configuration of phase shifting SMI system, where VT denotes an adjustable impedance and DAQ denotes . Terahertz level optical frequencies of laser cancel out due to self mixing leaving a relatively-stable photonic-microwave signal in observation. Inside dotted box is proposed signal processing containing carrier-separating and EMDWT.

Nanometer positioning accuracy PZT (P841,PI) has a movement range of 10um up to $400 \mathrm{~Hz}$ which is programmable for generating various vibration shapes. No electronic pre-processing circuits are used in this SMI. Dotted box of the configuration (seen in Fig.1) is main point of this proposal which describes a pure digital signal processing developed on Matlab software 
environment. Method of the improvement in the present system contains two main parts: 1. Use time-domain carrier-separating method to boost the demodulation speed. 2. Insert noise-elimination into intermediate processing not preprocessing to suppress the most kinds of noise source.

Acquired signal exemplified by Fig. 2 is inputted into signal processing to extract quadrature components of phase shift. The carrier-separating method not analyzes phase shift in each modulation period liking precious harmonics analysis [18]. Instead, quadrature signals are extracted sequentially by the filters, no resolution is lost and no amplitudes of higher harmonics are analyzed which allows directly extraction of quadrature signals with full bandwidth signal.

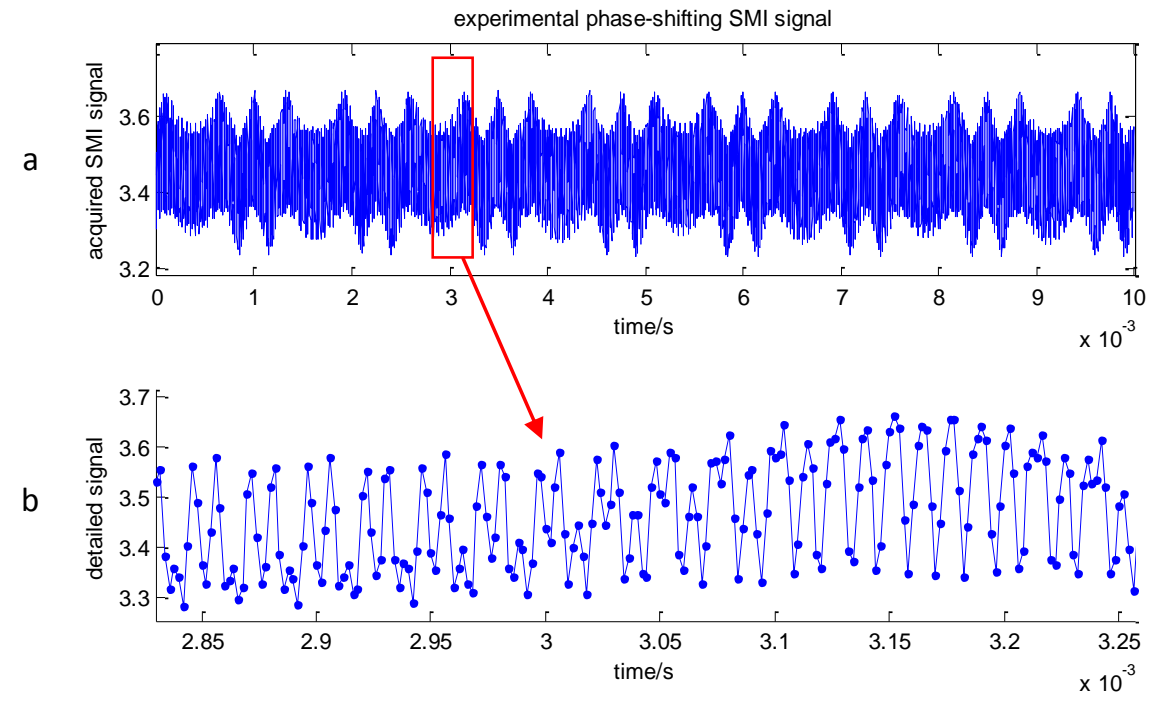

Fig.2. A typical phase shifting SMI signal contains numerous fringes lasting 10ms. Setup of DAQ is as follows: Sampling rate is set at $500 \mathrm{KHz} / \mathrm{s}$. Data point number is $5 \mathrm{~K}$. DAQ device adopts a digital TTL-triggered method to ensure initial phase of modulation waveform is zero. Vibration of PZT is sinusoidal with $410 \mathrm{~nm}$ amplitude at $400 \mathrm{~Hz}$ frequency. EOM parameters are: $40 \mathrm{KHz}$ modulation frequency, sinusoidal shape and $0.5 \mathrm{rad}$ depth.

\section{Method}

Based on theoretical studies of SMI [25], equivalent Fabry-Perot cavity of phase-shifted SMI (seen in Fig.3) is consisted of a complete-reflective mirror M1 and a compound mirror M23. In this paper, the effective reflection index of compound mirror depends on not only M2, M3 but also the phase shifting external optical length. Laser oscillation condition (Eqs.3) varies with the reflection index of M23. Therefore, the phase shifting optical length modulates the threshold stimulated gain of laser internal cavity and make laser intensity fluctuate to output the observed photonic-microwave signal.

$$
\begin{gathered}
r_{23}\left(f_{0}, \delta\right)=r_{2}+\left(1-r_{2}^{2}\right) r_{3} \exp \left(-j 4 \pi f_{0} \frac{\delta}{c}\right) \\
\delta=\Delta L+L+(\Delta n-1) d \\
\eta=\frac{r_{3}}{r_{2}}\left(1-r_{2}^{2}\right) \\
r_{1} r_{23} \exp \left(-j 4 \pi f_{0} \frac{n_{c} l}{c}\right) \equiv 1
\end{gathered}
$$


where $r_{23}$ stands for the reflection index of the compound mirror which is related to laser optical frequency and the effective external optical length. $\delta$ is the varying external optical length.$\eta$ is the coupling coefficient describing how much reflected light re-enter laser cavity. $r_{2}$ and $r_{3}$ are reflection indexes of mirror M2 and M3 respectively. $n_{c}$ is the complex refraction of laser working medium. $\mathrm{f}_{0}$ denotes the unperturbed laser optical frequency.

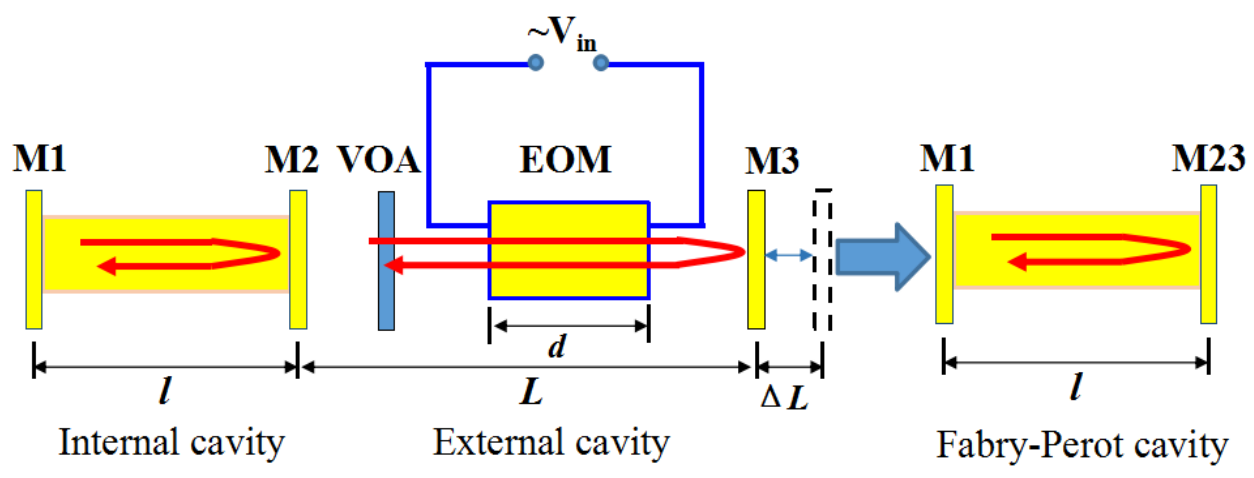

Fig. 3.Left side of this figure is three mirror model of sinusoidal phase-shifting SMI. Right side is the equivalent Fabry-Perot cavity where M23 is a virtual mirror with a varying reflection index.

Based on existing theories [25-27], the varied optical frequency is approximately expressed as:

$$
\mathrm{f} \approx \mathrm{f}_{0}-\frac{\eta \sqrt{1+\alpha^{2}} \sin (4 \pi \delta \delta / c-\arctan (\alpha))}{4 \pi \mathrm{m}_{0} l}
$$

$\mathrm{n}_{0}$ is real refraction index of laser medium. $\alpha$ is line-width enhancement factor determined by inherent characteristic of laser. Correspondingly, phase condition in angular frequency format is written:

$$
\begin{gathered}
\omega \mathrm{T}=\omega_{0} \mathrm{~T}-\mathrm{C} \sin (\omega \mathrm{T}-\arctan (\alpha)) \\
\mathrm{T}=\frac{2 \delta}{\mathrm{C}}
\end{gathered}
$$

where $\mathrm{C}$ denotes the feedback strength of reflected light and is defined as:

$$
C=\frac{T}{T_{\text {in }}} \eta \sqrt{1+\alpha^{2}}
$$

Phase modulation depth denotes amplitude of phase carrier. As suggested by Eq.2, phase modulation depth depends on the applied voltages and is precisely set at $0.5 \mathrm{rad}$ by adjusting the driven voltage amplitude. Considering that the laser beam passes through the EOM twice in one round trip, the phase modulation depth doubles. Then, the phase shifting SMI optical intensity is modified as:

$$
\mathrm{P}=\mathrm{P}_{0}\left[1+\operatorname{mcos}\left(\left(\varphi+\sin \left(\omega_{\mathrm{m}} \mathrm{t}\right)+\varphi_{0}\right)-\mathrm{C} \sin (\ldots)\right)\right]
$$

With:

$$
\varphi=\omega_{0} \mathrm{~T}
$$

(8a) 


$$
\varphi_{0}=\frac{4 \pi(\mathrm{L}-\mathrm{d})}{\mathrm{C}}
$$

(8b)

$\mathrm{m}$ is the undulation index.$P_{0}$ is the direct component. $\varphi_{0}$ stays constant denoting the unchanged physical external cavity length. For our purpose, the extreme minimum Csin(...) are omitted when SMI occurs at weak feedback region [28]. Following the Eqs. 1 and 2, driven voltage to EOM is adjusted to set phase modulation depth at 0.5rad for separating the modulation carrier, the laser intensity neglecting the higher feedback takes the below form:

$$
\mathrm{P} \approx \mathrm{P}_{0}\left[1+\operatorname{mcos}\left(\varphi+\sin \left(\omega_{\mathrm{m}} \mathrm{t}\right)\right]\right.
$$

$\omega_{\mathrm{m}}$ denotes the angular frequency of sinusoidal carrier. In previous works [16-19], Eq.8 was often expanded in Bessel function series and analyzed FFT, inverse FFT or Z-transform in frequency domain. To avoid complicated analysis, this section expands the phase-shifting SMI signal in simple trigonometric functions:

$$
P=P_{0}+m P_{0} \cos (\varphi) \cos \left(\sin \left(\omega_{m} t\right)\right)-m P_{0} \sin (\varphi) \sin \left(\sin \left(\omega_{m} t\right)\right)
$$

Without Bessel functions, Eq.10 contains two alternating components where $\sin \left(\omega_{\mathrm{m}} \mathrm{t}\right)$ is considered as the phase carrier. Obviously, $\sin (\varphi)$ and $\cos (\varphi)$ are a couple of quadrature signals contained in the two alternating components. The tricks of the carrier-separating method are explained subsequently with FFT analysis. We multiply the phase shifting SMI signal with the sine function of carrier and expressed as:

$$
P \times \sin \left(\sin \left(\omega_{m} t\right)\right)=-m P_{0} \sin (\varphi) / 2+P_{1}+P_{2}+P_{3}
$$

With:

$$
\begin{gathered}
P_{1}=P_{0} \sin \left(\sin \left(\omega_{m} t\right)\right) \\
P_{2}=m P_{0} \cos (\varphi) \sin \left(2 \sin \left(\omega_{m} t\right)\right) / 2 \\
P_{3}=m P_{0} \sin (\varphi) \cos \left(2 \sin \left(\omega_{m} t\right)\right) / 2
\end{gathered}
$$

$P_{1}, P_{2}$ and $P_{3}$ in frequency domain concentrate at integer times of modulation frequency as shown by Fig.4.

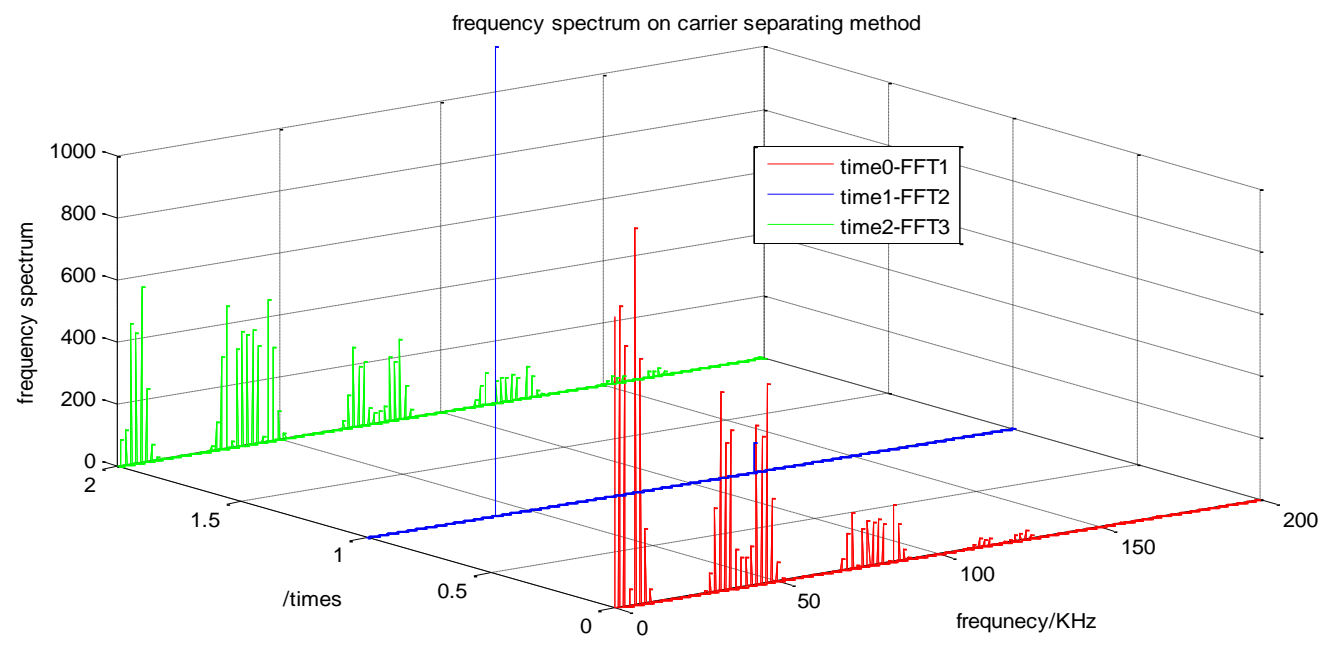


Fig. 4. Time-axis denotes the order of implementing fast Fourier transform. Frequency-axis denotes frequency-domain range. Spectrum-axis denotes power spectra obtained by FFT. Time0-FFT1 is acquired phase shifting SMI signal containing abundant higher-order harmonics in frequency domain. Time1-FFT2 is sine function of phase carrier in frequency domain concentrating at $40 \mathrm{KHz}$ modulation frequency with narrow bandwidth. Time2-FFT3 is Eq.11 in frequency domain, where energy of fundamental wave and harmonics is re-distributed by the multiplication calculation and harmonic components still center around the integer times of modulation frequency.

Since modulation frequency and sampling data points are known beforehand, computer can easily generate the sequential data points of $\sin \left(\sin \left(\omega_{\mathrm{m}} t\right)\right)$ and $\cos \left(\sin \left(\omega_{\mathrm{m}} \mathrm{t}\right)\right)$ at same sampling rate of collected experimental signal. Based on frequency spectral characteristic, phase carrier $\sin \left(\sin \left(\omega_{\mathrm{m}} \mathrm{t}\right)\right)$ is separated from $\sin (\varphi)$ by multiplication. In addition, direct bias of laser power is shifted into $P_{0} \sin \left(\sin \left(\omega_{\mathrm{m}} \mathrm{t}\right)\right)$. Beneficially, $-\mathrm{mPsin}(\varphi) / 2$ distributes at the lowest frequency part, therefore, $-\mathrm{mPsin}(\varphi) / 2$ can be filtered out easily by a common low pass filter:

$$
m P \sin (\varphi) / 2=-L P F\left(P \times \sin \left(\sin \left(\omega_{m} t\right)\right)\right.
$$

Similarly to above extraction, SMI signal is multiplied with cosine function of phase carrier and low-pass filtered to obtain the $\mathrm{mPcos}(\varphi) / 2$ :

$$
\mathrm{mP} \cos (\varphi) / 2=\operatorname{LPF}\left(\mathrm{P} \times \cos \left(\sin \left(\omega_{\mathrm{m}} \mathrm{t}\right)\right)\right.
$$

Amplitudes of Eqs.13 and 14 are identical and are wiped off by arc-tangent calculation:

$$
\varphi=\operatorname{unwrap}\left(\tan ^{-1}\left(\frac{\mathrm{Eq} .13}{\mathrm{Eq} .14}\right)\right)
$$

Considering that direct component is shifted to a higher frequency zone, no high pass filter is required like the works of Ref. [19]. Therefore, the Eqs.9-14 describe mathematical process of carrier-separating method and are implemented on the acquired sample signal to extract phase-shift (seen in Fig.5). Two quadrature signals in frequency domain have similar bandwidth.

Reconstructed phase has same data points with acquired experimental signal, which means the full bandwidth of the dataacquisition is utilized using carrier-separating method and permits for a larger dynamical measuring range. In conclusion, the Eqs.9-14 provides a simple flexibility to extract quadrature signals on condition that the phase modulation depth equals $0.5 \mathrm{rad}$. Following the linear relationship between phase and vibration, $8.1 \mathrm{rad}$ phase corresponds with $407.8 \mathrm{~nm}$ in retrieved vibration. If adding noise-elimination operation, the deviation is narrowed to $1.3 \mathrm{~nm}$ level. The time complexity of carrier-separating method is simpler than Bessel or FFT analysis because the algebraic operations cost less time than integration calculations. Execution of Matlab codes of the carrier separating method takes only 10 milliseconds to finish whole analysis. Since the collected signal lasts only 10 milliseconds, over forty times demodulation are finished per-second. Undoubtedly, the same method programmed 
on DSP (digital signal processing) will be extremely high efficiency which is specifically suitable for real-time demodulation.
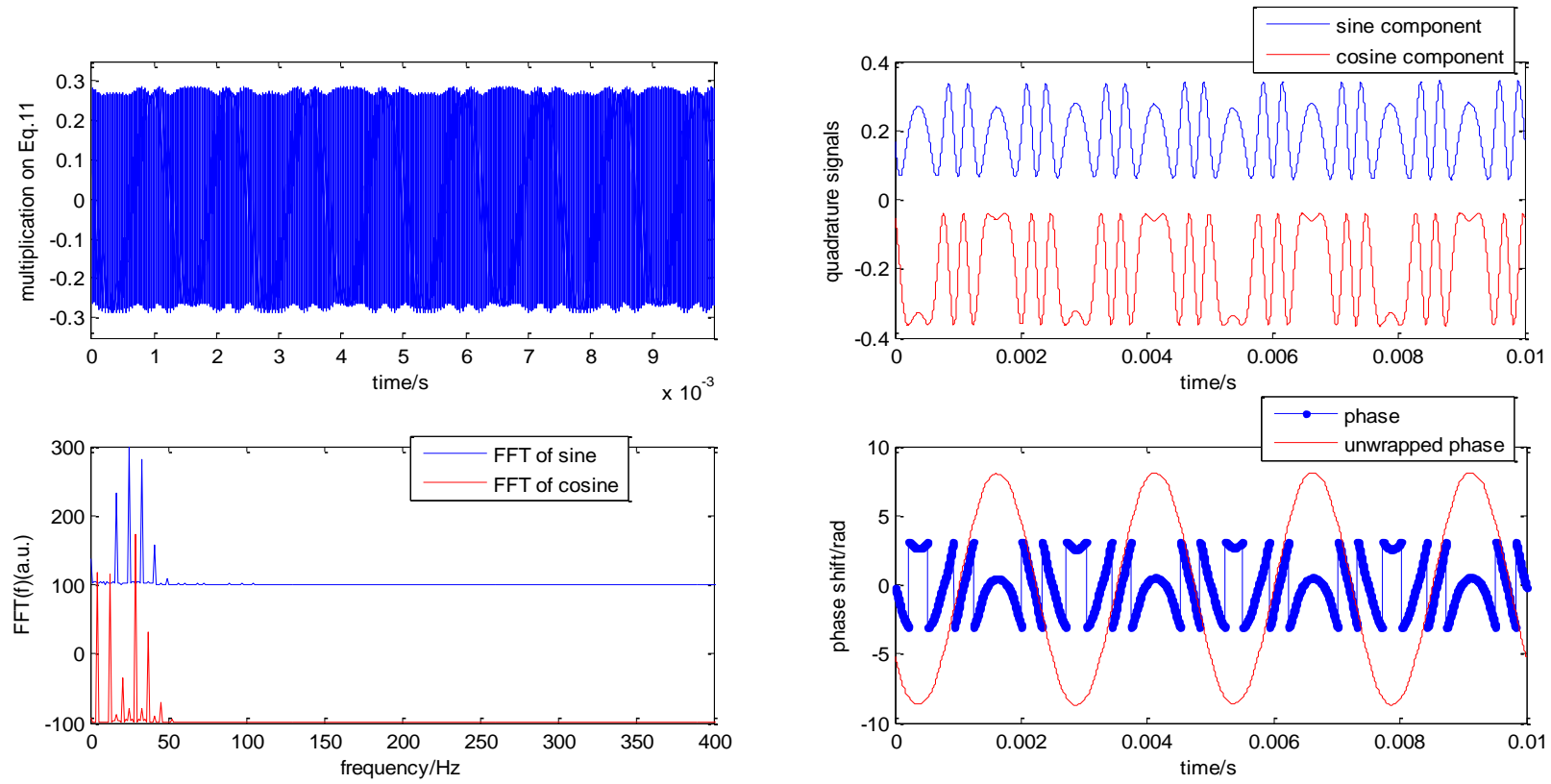

Fig. 5. (a). Multiplication(Eq.11) on the collected signal(Fig.2a) and sine function of sinusoidal phase carrier, the frequency spectra of this multiplication is time-2 FFT3 of Fig.4. (b) Two extracted quadrature signals lasting 10ms(Eqs.13 and 14) output by two LPFs, where sine component is directly filtered out from Fig.5a. (c) Extracted quadrature signals in frequency domain with same bandwidth. (d) Phase shift figured out by arc-tangent(Eq.15) calculation which fluctuates between $-\pi$ and $\pi$. After unwrapping, the sequential phase curve mirrors the measured vibration.

\section{EMDWT}

Noises of the established laser system can be generally classified into four kinds: the electronic, the thermal, the optics and the mechanics. Firstly, electronic noise results from driving voltages and I/V conversion. Although digital DDS sinusoidal signal generator and a precise resistor are adopted in experiment, ripples of applied voltages on EOM induces a nonlinear error. The instantaneous measurement error related to the noise floor of EOM is up to 0.0025rad since EOM electronics has a root mean square noise of near $100 \mathrm{mV}$. This slight noise of induced phase is evaluated by below Eqs. 16 to19:

$$
\begin{aligned}
& P^{\prime}=P_{0}+m P_{0} \cos (\varphi) \cos \left(\sin \left(\omega_{m} t\right)+\Delta \alpha\right) \\
& -m P_{0} \sin (\varphi) \sin \left(\sin \left(\omega_{m} t\right)+\Delta \alpha\right)
\end{aligned}
$$

Repeating the carrier-separating method on the noised expression, we get:

$$
\begin{aligned}
& P^{\prime} \times \sin \left(\sin \left(\omega_{m} t\right)\right)=P_{0} \sin \left(\sin \left(\omega_{m} t\right)\right)+ \\
& m P_{0} \cos (\varphi) \sin \left(2 \sin \left(\omega_{m} t\right)\right) \cos (\Delta \alpha) / 2 \\
& -m p\left(1-\cos \left(2 \sin \left(\omega_{m} t\right)\right) \cos (\varphi) \sin (\Delta \alpha) / 2\right. \\
& +m P_{0} \sin (\varphi)\left(1-\cos \left(2 \sin \left(\omega_{m} t\right)\right) \cos (\Delta \alpha) / 2\right. \\
& +m p \sin \left(2 \sin \left(\omega_{m} t\right)\right) \sin (\varphi) \cos (\Delta \alpha) / 2
\end{aligned}
$$

With the quadrature signals become:

$$
\begin{aligned}
& \operatorname{LPF}\left(P^{\prime} \times \sin \left(\sin \left(\omega_{m} t\right)\right)\right)=m P_{0} \sin (\varphi) \cos (\Delta \alpha) \\
& -m p \cos (\varphi) \sin (\Delta \alpha) / 2
\end{aligned}
$$


(18b)

With $\Delta \alpha=0.0025 \mathrm{rad}$, so we can get a relative amplitude ripple is less than one percent:

$$
\sin (\Delta \alpha) \approx 0.0024
$$

Secondly, the thermal noise shows as the laser temperature shift. Since laser only outputs1mw power, ninety percent of supplied electronic power turns into "waste heat" which rises the ambient temperature of laser tube. If without Peltier cooling devices, the ascending temperature influences the demodulation notably. Thermal noise can not only happen inside of laser tube but also in driven electronic circuits and degrades the performance of driving waveform too. Correspondingly, an effective air-conditioner is kept working in the closed experimental room where the temperature fluctuation is limited within two degrees.

Thirdly, various optical noises exist in the laser system, the mistaken alignment, unsuitable feedback level, instability of laser optical frequency and varying air refraction index of external cavity. According to the updated Edlen formula [29], the air refraction index suffers from temperature, pressure and humidity and induces an unwanted pseudo optical length change.

$$
\delta \mathrm{n}_{\mathrm{air}}=[0.00268 \delta \mathrm{P}-0.929 \delta \mathrm{T}-0.00042 \delta \mathrm{f}] \times 10^{-6}
$$

where $\delta P, \delta T$ and $\delta f$ denote the variation of air pressure, temperature and humidity respectively. However, the magnitude of refraction index change due to environmental disturbance of experiment room is at 10e-6 level, which means the refraction index variation induces a pico-meter level uncertainty to micron vibration measurement. Based on laser physics of single mode 632.8nm He-Ne laser [26][30], optical laser frequency[31] is a terahertz quantity with a stable short-time uncertainty. Therefore, the relative variation of wavelength $\Delta \Lambda$ is about 10e-7 level. Speckle noise rooting from rough target surface is another optical noise and will degenerate the measurement[31]by inducing a pseudo vibration. Two factors in experiment determine the speckle noise level: diffusive angle of emitted laser beam and area size of laser beam projecting on target [33-35]. In real situation, diffusive angle of He-Ne laser is extremely small less than 3mrad. Fortunately, laser beam is kept at single linearly polarization due to installed Brewster window inside laser tube, the reflection area on target is limited in a highlight point. SMI exists at single linearly polarization due to the Brewster window installed in cavity, therefore, the atom gas He-Ne laser offers a natural insensitivity to optical speckle noise, as a result, speckle phase error is weak, $\Delta \varphi_{\text {speckle }} \ll 0.1$.In SMI process, feedback level directly effects shape of fringes and produces anomalous spectral broadening. Therefore, the feedback should be finely controlled at weak level.

At last, lateral vibration of PZT and non-longitudinal vibration of platform induce pseudo vibration into SMI as mechanical noise. Besides, open-loop of PZT causes unusual fluctuation of the measured phase shift. With improved design on mechanics, the mechanical noise will decrease as well. Above four kinds of noise appear simultaneously in SMI and transfer randomly error to reconstructed phase shift, the possible error is estimated via the following relation:

$$
\text { Error }=\sqrt{\sin ^{2}(\Delta \alpha)+\delta \mathrm{n}_{\text {air }}{ }^{2}+\Delta \Lambda^{2}+\Delta \varphi_{\text {speckle }}{ }^{2}}
$$

Using Eq.21, the possible error in laboratory environment is about $10 \mathrm{~nm}$ and agrees with experimental results. Besides, the permanent external cavity length induces a constant initial phase-shift in measurement which effects the result when vibration amplitude is very small. However, when either amplitude or frequency of measured vibration is big enough, permanent phase shift can be neglected or offset.

Due to the existence of above mentioned noises, an effective noise-elimination operation is important and favorable for improving the signal-to-noise ratio. In this paper, we combine the advanced EMD decomposing algorithm with continuous WT 
to enhance useful self-mixing fringes. EMD is a powerful tool for analyzing non-linear time signal by decomposing a time series into a finite and small number of Intrinsic Mode Functions (IMF) which has been described elsewhere [40]. The basic operations of EMD are as follows:1. EMD identifies all extrema points in digital signal and interpolates between minima points to form a low envelop, then interpolates between maxima points to get an upper envelop. 2 Calculates mean values of the upper and low envelops and subtracts the values from extrema points to get a IMF component. 3. Removes the obtained IMF component from signal to form a residual, then identifies a new group of extrema points from the residual and repeats the above steps 1 and 2, until last extrema points are constant or monotone function. $\mathrm{mPsin}(\varphi) / 2$ is rewritten by EMD as below:

$$
\operatorname{mPsin}(\varphi) / 2=\sum_{i=0}^{N} \operatorname{IMF}(\mathrm{i})+\text { residual }
$$

where $\mathrm{N}$ is the IMF number and $\mathrm{i}$ is positive integer up to $\mathrm{N}$. IMFs reflect all frequency components of $\mathrm{mPsin}(\varphi) / 2$ from high to low(seen in Fig.6). Decomposition procedure is data-driven and decides the level automatically. In experimental signal, the slow temperature shift corresponds with the lowest IMF, high-frequency electronic noise corresponds with highest frequency IMF which are easy to remove. The other IMFs are rreassembled and normalized:

$$
\left.\sin (\varphi)=2 \sum_{i=1}^{N-1} \operatorname{IMF}(i)\right) / m P
$$

Above superposition of IMFs has a narrower frequency bandwidth and is a more stationary signal, then, recombined signal is inputted to continuous wavelet transform(CWT) subsequently for further noise-elimination.
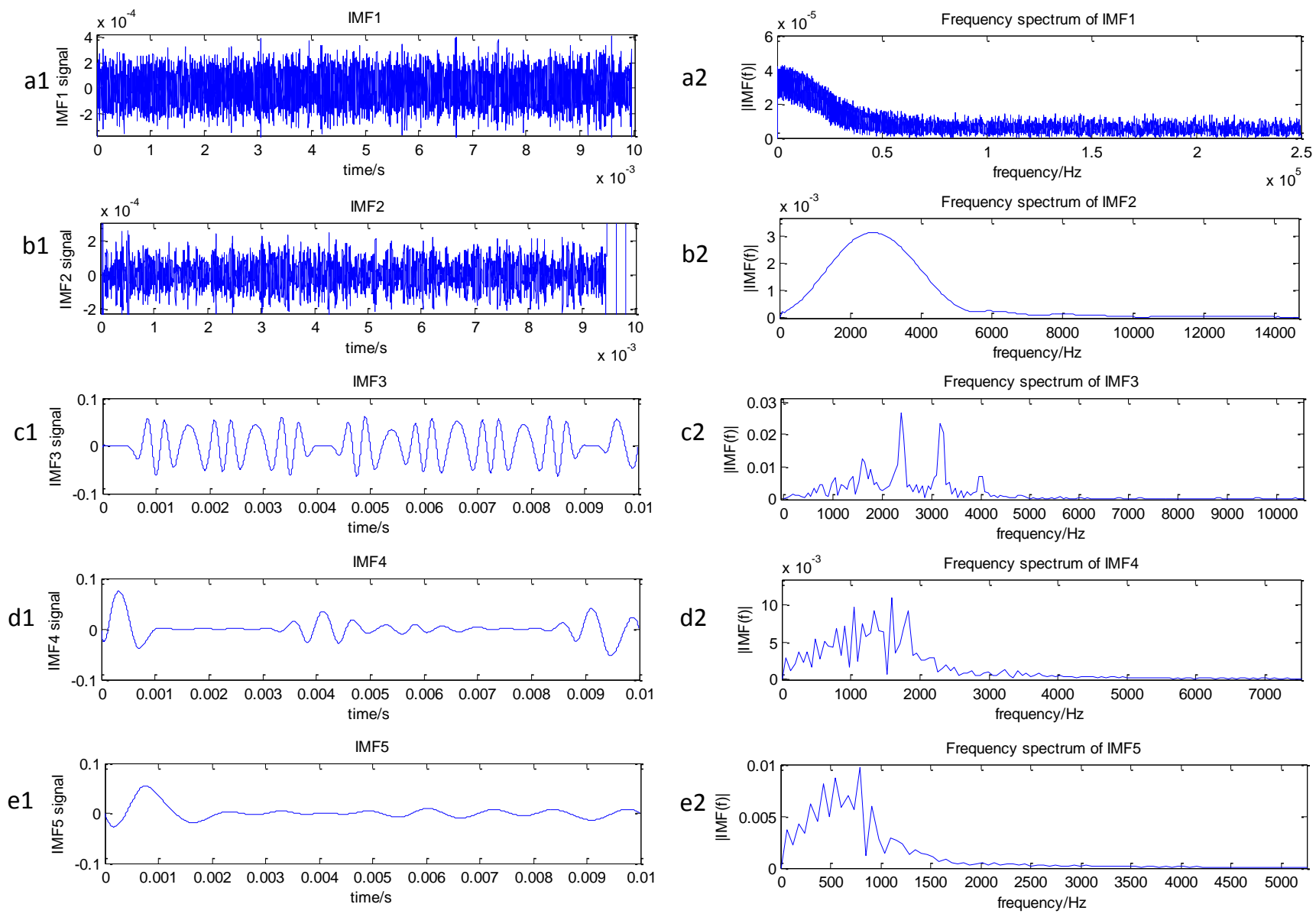
Fig. 6. IMFs(seen in a1 e1) decomposed by EMD on extracted-mPsin $(\varphi) / 2$ and their corresponding frequency spectrums (seen in a2 e2) from high to low. In following decomposition process, highest and lowest frequency IMFs is out of consideration.

To suppress unwanted noises, conception of CWT is intended here by assistance of EMD and serves as a powerful tool of eliminating strength noise[37]. According to existing application of CWT[36], CWT provide information of spectrum changes along time-axis in time-frequency joint domain analysis and is a natural filter to high-frequency components. Specially, carrierseparating demodulation is a simplified phase demodulation offering a much better accuracy than fringe-counting[38] and is realized in pure time-domain. CWT implemented on obtained sine component of quadrature signals is for decomposing the noises out and takes the below form:

$$
\mathrm{CWT}(\mathrm{Db}, \sin (\varphi))=\mathrm{a}^{-12} \int \sin (\varphi) \bar{\psi}\left(\frac{\mathrm{t}-\mathrm{b}}{\mathrm{a}}\right) \mathrm{dt}
$$

where coefficients denotes the similarity degree between wavelet and Eq.22. a denotes the time-scaling function, describes the time length of one single period basic wavelet, which increases gradually in decomposition process and is inversely proportional to the frequency component of the analyzed signal.b denotes the shift operator, which denotes the shifting of wavelet in time-axis. $\bar{\psi}$ is complex conjugate format of $\psi$. As a is decreased,the wavelet in the time domain is extended, and its corresponding spectrum is narrow. Meanwhile, $b$ is shrunk in time, thus, decreasing time-scaling function will gradually obtain approximate low-frequency component of SMI signal, which is called as decomposing process.This makes the wavelet transform a unique filter to suppress high-frequency noises with SMI signal less changed than common filters.

Existing wavelet type can be a host of types: Daubechies(db), Morlet, Coiflets, Symlets, Haar, Biorthogonal, Hat and Meyer and their subsets. However, not all wavelets are suitable for decomposition process. Choosing one of them depends upon the application and nature of the signal. For example, Morlet wavelet enables researcher to extract robust fringes and amplitude from the time envelope of SMI signal[39], however, phase demodulation method focuses on the phase-shift induced by SMI. Instead of fringes, the phase shift is parameter of interest in this signal processing and need be kept as complete as possible. Meanwhile, both speckle noise and feedback level is weaker than laser diode sensors, optical intensity noise induced by above noise sources are main objective to be eliminated. Therefore, Daubechies series wavelet is proved (seen in Fig.7) to be more suitable than Morlet wavelets to maintain useful phase shift. In conducted comparison decomposition process, the fourth level decomposed approximate coefficients by db10, bior1.5 and haar wavelet are different. Only coefficient by db10 is selected to recover an enhanced sine component(not fringes) on below inverse wavelet transform:

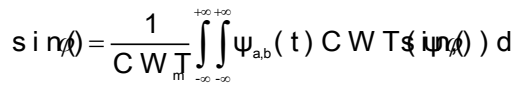



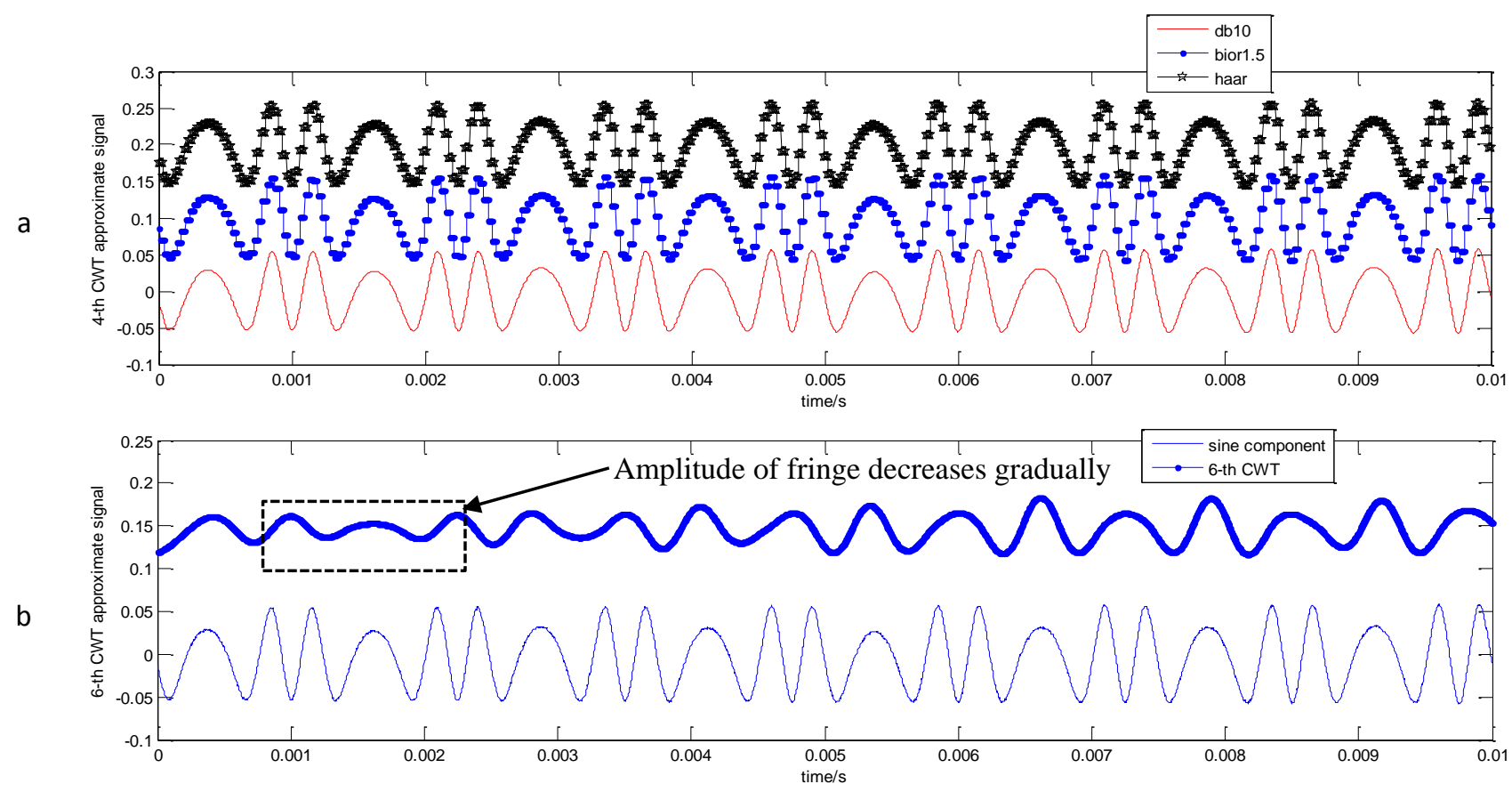

Fig.7.(a)The fourth approximate signals by CWT using three basic wavelet type:db10, bior1.5 and haar, where approximate signal by db10 wavelet show more sooth waveform than other two types. (b) The sixth approximate signal and initial sine component (result of Eq. 23). Compared with sine component, amplitude of decomposed fringes using db10 wavelet gradually decreases with increasing of decomposing level in dotted box, therefore, decomposition process should end at the fifth level.

Another problem for CWT decomposition is how to determine the decomposing level, although high-frequency noise is gradually decreased by decomposition by CWT, useful phase information contained in fringes is decreased until vanishing. Seen from Fig.7b, amplitude of useful fringe is weakened in dotted box and form incomplete fringes which is incorrect for following arc-tangent calculation. To address this problem, fringes counting operates with a comparator to count an effective fringe numbers in each decomposed approximate signal(seen in Fig.1), which is for determine the ending level. Taking Fig.7 as example, sine component is decomposed four level using Db10 wavelet and recovered by Eq.25 before arc-tangent calculation. When amplitude of vibration is smaller than half wavelength, fringe counting will failed and decomposition should stops at 3rd level. EMDWT is designed to decompose random noise out from quadrature signals not from the collected phase-shifting SMI signal. It can be found total decomposing level never exceeds 20, which adds a little time expense to signal processing.

\section{Comparison}

In our experiment, sine and cosine functions of phase carrier are utilized to extract the quadrature components of phase shift, $0.5 \mathrm{rad}$ phase modulation depth is a required condition of implementing phase carrier separating method. Objective mirror mounted on the PZT is measured by both the SMI and PloyTec-5000 laser Doppler velocity meter with same vibrations in the same laboratory room as verification. Some more experiments are conducted to further test the signal processing where the frequency-varying, damping and nanometer-level vibrations are measured.

Mechanism of LDV is different to phase-shifting SMI and uses heterodyne interferometer principle. LDV in comparison has slim optical fiber cable and a flexible fiber-coupled sensor head for inaccessible measurement points. Advanced hardware extracts the velocity by frequency demodulation precisely with a resolution better than 1nm (illustrated by Fig.7). A 40MHz carrier is generated on its photo detector with the aid of a Bragg cell in LDV. However, EOM in proposed SMI system has a broad operating bandwidth up to $0.25 \mathrm{GHz}$ offering a higher frequency carrier.

In respect to laser source, LDV employs a possible one or maximum of two modes gas laser, depending on the laser cavity length. If the gas laser works with two modes, interference effects will cause the intensity of the resulting optical signals to vary periodically with the stand-off distance. While, the proposed system employs a single mode laser, SMI signal is independently 
of the stand-off distance. As the role of thumb to our method, sine and cosine functions of phase carrier are separated precisely only when 0.5 rad phase modulation depth is kept unchanged during experimental measurements. In experiment, objective mirror is mounted on PZT and measured by both SMI system and Ploytec-5000 laser Doppler velocity meter with same parameters in laboratory room as verification.

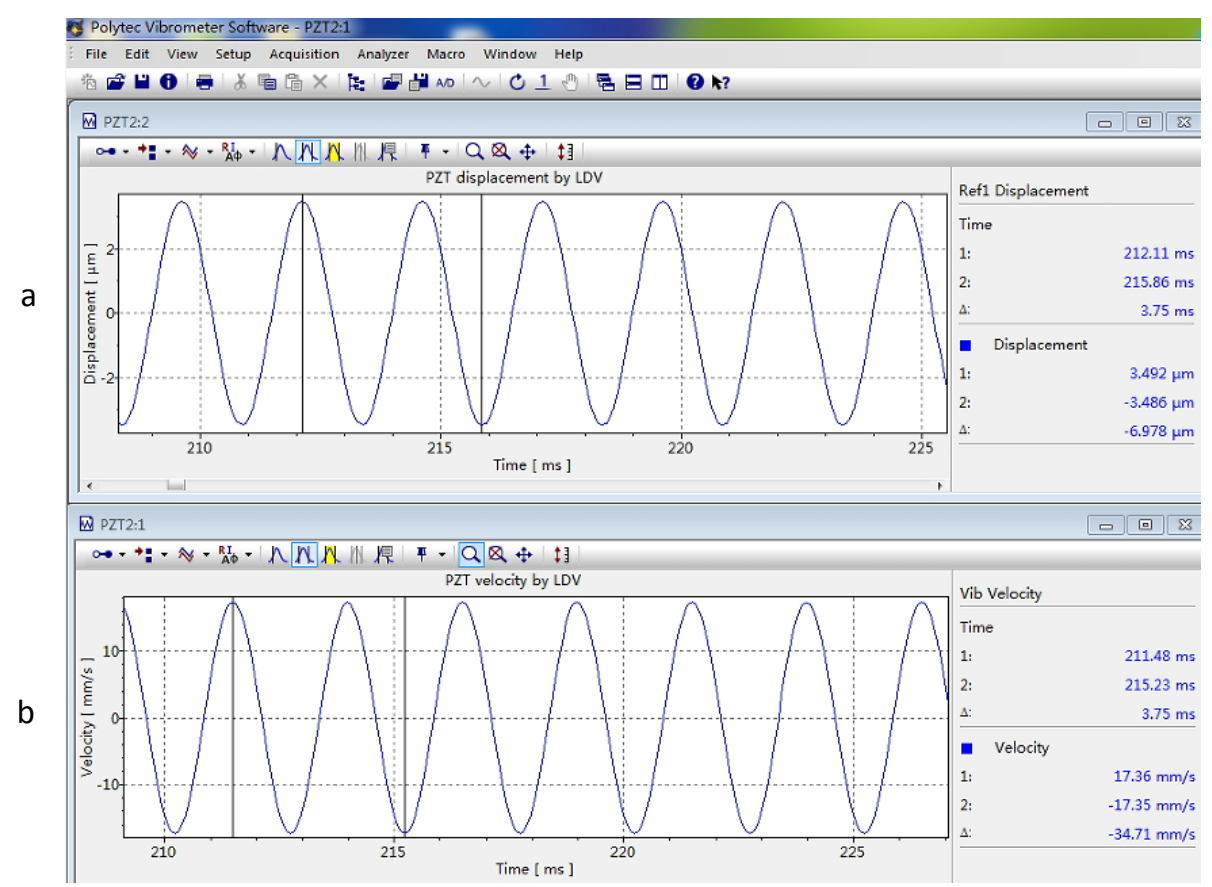

Fig. 8. Typical measurement results by Polytec-5000 laser Doppler velocity meter by screen-shot with a resolution of $1 \mathrm{~nm}$. (a). The displacement obtained by LDV with peak-to-peak amplitudes are $3.492 \mathrm{um}$ and $-3.486 \mathrm{~nm}$. (b) The velocity curve by LDV with peak amplitude is $17.36 \mathrm{~mm} / \mathrm{s}$. The tested PZT is set to vibrate at $400 \mathrm{~Hz}$ with $3490 \mathrm{~nm}$ by PI software, average amplitude measured by SMI is $3489 \mathrm{~nm}$.

The linear relationship between phase-shift and the measured vibration is unchanged, arbitrary vibrations can be converted by extracted phase-shift. As verification, random vibrations are measured and compared in Fig.9.Results of two instruments are plotted together as comparison for testing feasibility (seen in Fig.9). Correlation coefficients of Pics. 9b and 9c are 99.95\%and $99.93 \%$, respectively. Average deviation $\Delta \zeta$ and standard deviation SD of two instruments are calculated on below relationship:

$$
\begin{aligned}
& \Delta \zeta(\mathrm{i})=\frac{\sum_{\mathrm{i}=0}^{\mathrm{i} M} \Delta \mathrm{L}_{\text {suI }}(\mathrm{i})-\Delta \mathrm{L}_{\text {LDV }}(\mathrm{i})}{\mathrm{N}-1} \\
& \mathrm{SD}=\sum_{\mathrm{i}=1}^{M} \sqrt{\frac{\left(\Delta \mathrm{L}_{\text {sII }}(\mathrm{i})-\Delta \mathrm{L}_{\text {LV }}(\mathrm{i})\right)^{2}}{\mathrm{~N}-1}}
\end{aligned}
$$

where $M$ denotes data point number, $\Delta \mathrm{L}_{1}$ and $\Delta \mathrm{L}_{2}$ denote vibrations generated by SMI and LDV respectively. $\Delta \zeta$ of Fig.9a is $1.8 \mathrm{~nm}$ with SD $=1.21 \mathrm{~nm}$. Due to EMDWT, most noises vanish away in signal processing, $\Delta \zeta$ of Figs.9b and $9 \mathrm{c}$ are $1.9 \mathrm{~nm}$ and 1.6nm. Discrepancy between two different principle instruments may stem from the electronic jitter of PZT controller or misalignment of Polytec-5000. When laser beam from fiber-coupled laser of LDV is not coinciding with optical longitudinal axis of SMI, error of two results is influenced by the angle deviation between two optical paths. The short-term resolution, told by the results, is about $1.5 \mathrm{~nm}$ between two instruments. If optics mechanical design can be modified for reducing the alignment deviation, error will be further decreased. 

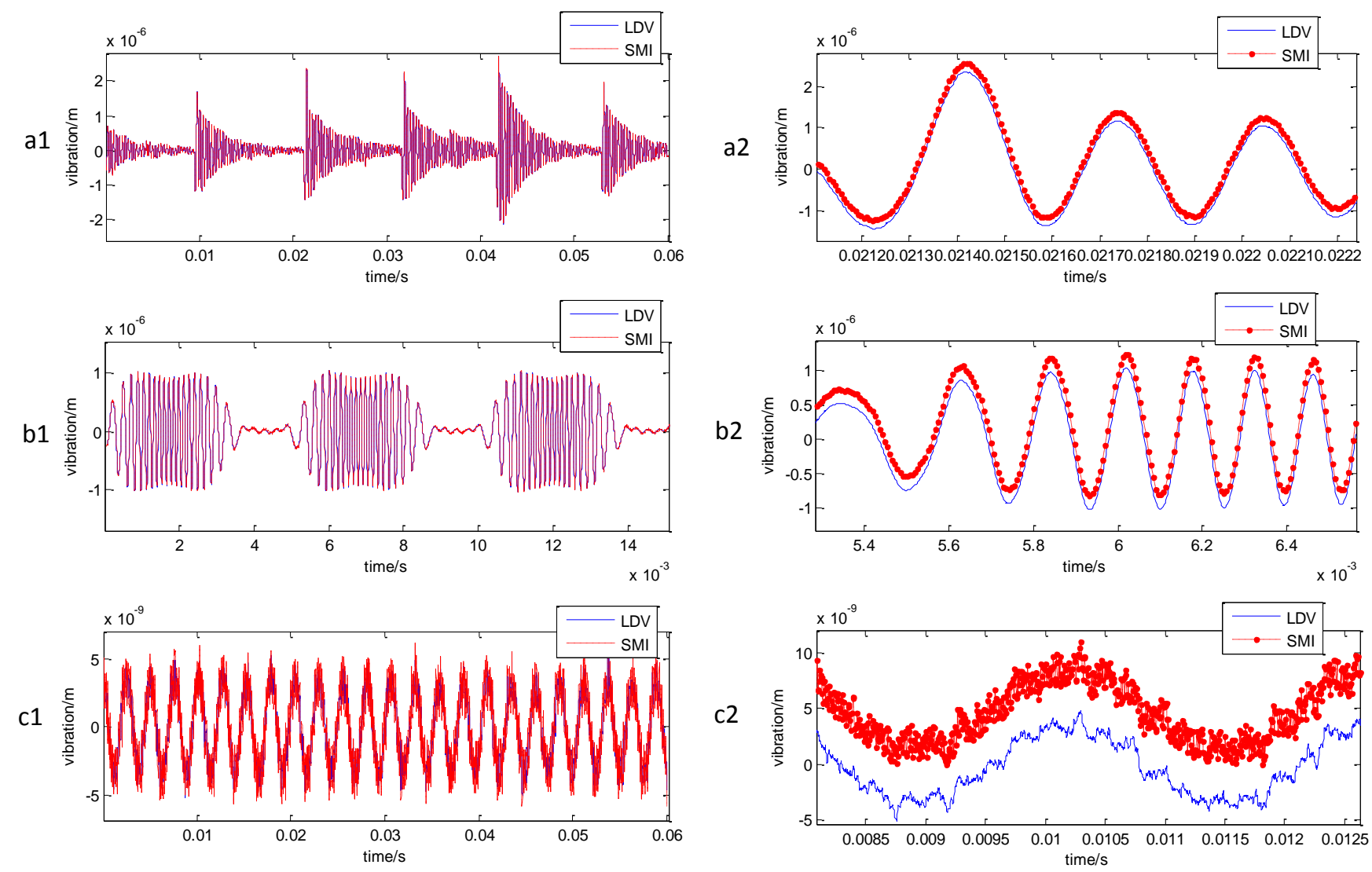

Fig.9. Random displacement obtained by two instruments, red point and black curves are from SMI and LDV, respectively. (a1). Random damping vibration realized by slightly knocking the PZT at longitudinal direction lasts 30ms. (a2). Fraction of measured random damping vibration by two instruments lasting about $0.8 \mathrm{~ms}$. For convenience observation, the SMI result is biased 200nm. (b1).Burst vibration measured by two instruments where SMI results is biased 200nm. (b2). Fraction of Fig.9b1. (c1).Closed-loop 4nm damping vibration at 20Hz. (c2). Fraction of 4nm damping vibration, where SMI results are biased 5nm.

The second purpose of improvement is to employ noise-elimination in intermediate signal processing to enhance the signal feature. Therefore, the measurement of harmonic vibration is repeated for collecting amplitudes to evaluate the repetitive error ranges(seen in Fig.10). In theory, if modulation frequency is $500 \mathrm{KHz}$ and self-mixing fringe frequency is only $5 \mathrm{~Hz}$, a period of half wavelength displacement is subdivided into $100 \mathrm{~K}$ sections, therefore, theoretical resolution is $N / 200000$, about $3.2 \mathrm{pm}$, which is impossible in reality by any phase demodulation methods. Even being shut down,stationary PZT has a random position change within several hundreds of picometers. As a result, the repetitive measuring error is proved with limitations of experimental devices: as to this laser system, only in measurement of micron-level vibration(less than $2 \mathrm{um}$ ), modulation frequency must exceed $40 \mathrm{KHz}$ and used laser is typical linearly polarized at weak feedback level. In given results, variance is defined as the deviation between two measurement results of amplitudes, which contains the system error floor of SMI and angle deviation between two instruments. In order to examine the repeatability, measurements of three groups of vibration amplitude are performed and compared. Seen from collected error bars, upper and lower variances of measured amplitude are less than 2nm with EMDWT, while individual carrier-separating gives an maximum variance of 10nm, showing proposed method can be applied to measure dynamical vibration with high sensitivity. 
a

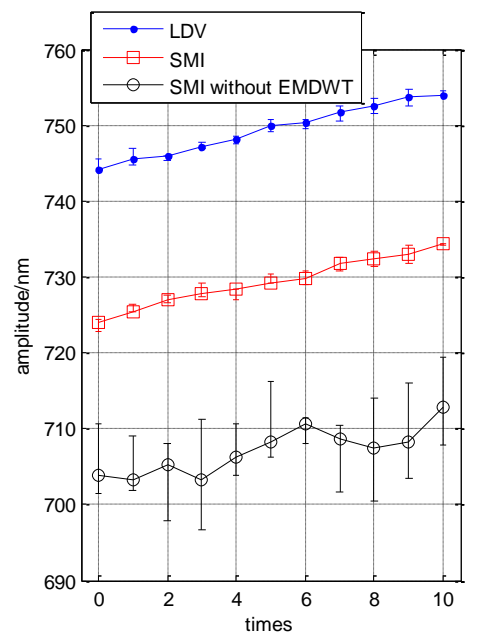

b

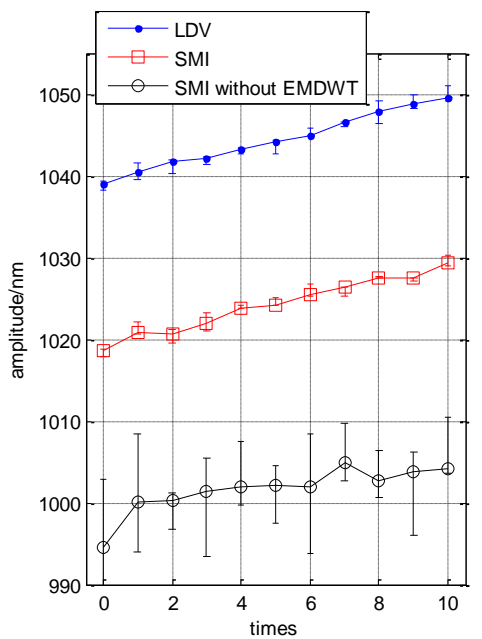

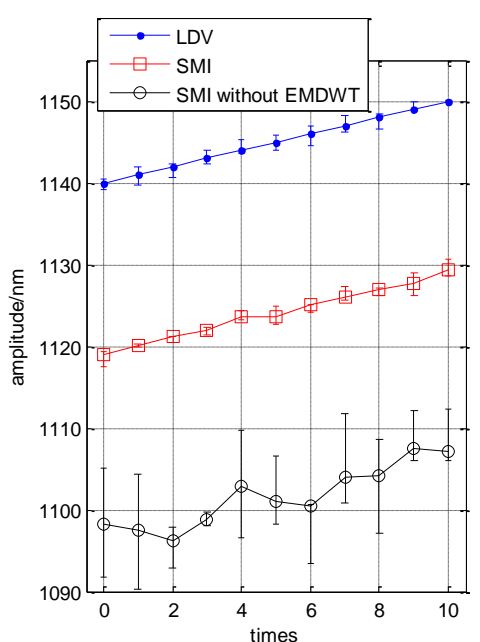

Fig. 10. (a). Short-term(measurement is finished in 20 minutes) average amplitudes on $20 \mathrm{~Hz}$ sinusoidal vibrations measured by two instruments where the amplitude increases from 705 to $715 \mathrm{~nm}$. SMI and LDV results are biased 20nm and 40nm respectively for distinguishing. (b) results of two instruments when amplitude ranges from 1000 to1010nm.(c) results when amplitude ranges from $1100 \mathrm{~nm}$ to $1110 \mathrm{~nm}$. The variance of results obtained from SMI without any noise-elimination, told by this figure, is about 9nm and decrease obviously if with EMDWT.

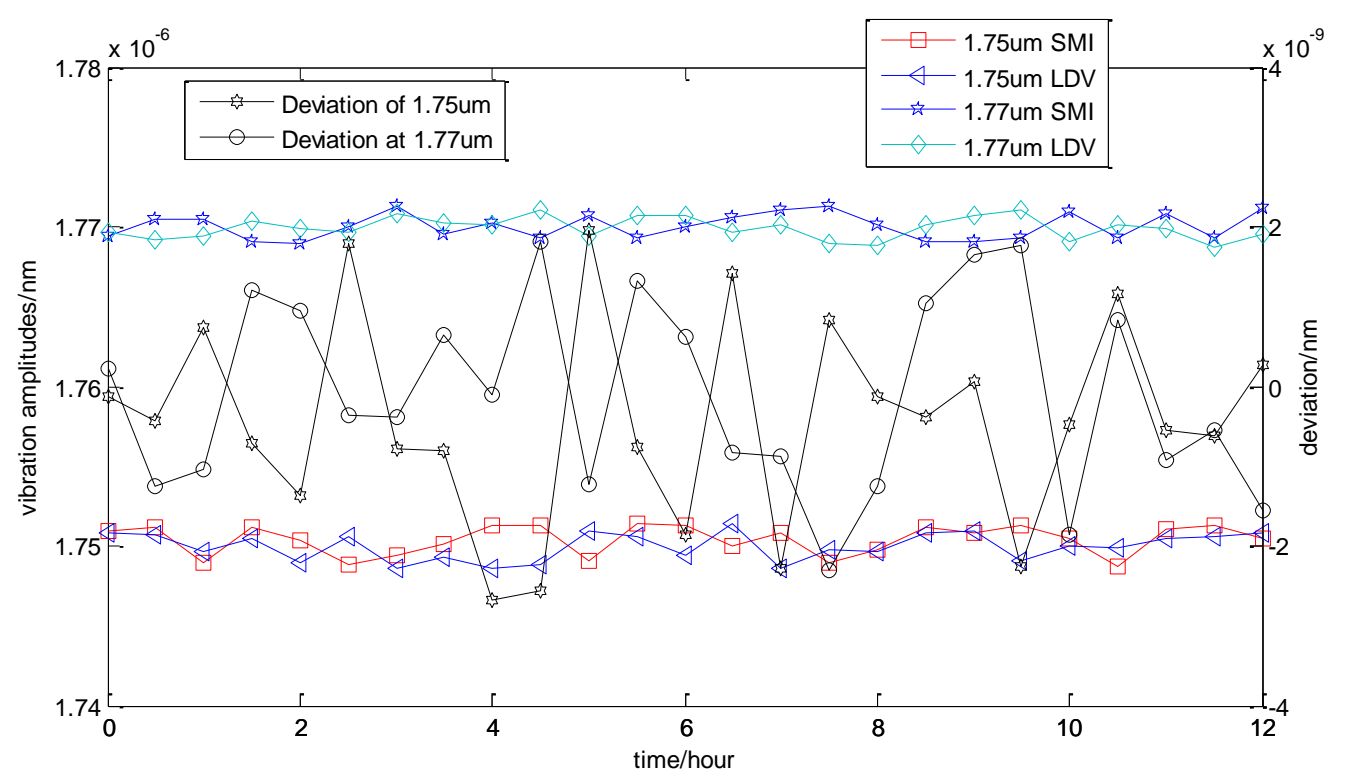

Fig. 11.Long-term average amplitudes measured in twelve hours at interval of half hour by two instruments and their deviations.

In long term, dual-output gas laser consumes low power with a narrow line-width, laser wavelength without feedback light has a little shifting $(<0.01 \mathrm{~nm})$ by spectroscopy with stable ambient temperature. To examine the long term performance with proposed signal process, SMI system is preheated for forty minutes and then is kept on running twelve hours in laboratory environment. At each half an hour, 1.75um and 1.78um damping vibrations on precision $\mathrm{PZT}$ at $10 \mathrm{~Hz}$ are measured by two instruments as typical samples and their average amplitudes are recorded (seen in Fig.11), where deviation of two results is less than $3 \mathrm{~nm}$. Like the short term measurement, the experimental results of two instruments are plotted simultaneously.

Continuous development of nano-technology pays more and more attention to dynamical measurement of vibration, which provides an ideal place for applying phase-shifting SMI. As demonstrated, proposed carrier-separating with EMDWT is the 
optimal choice for real-time extracting phase-shift due to its inherent advantages: EOM crystal introduces small insertion optical loss to SMI. This SMI is a kind of remote approach without damaging target. For practical applications, a rough target is better than cooperative mirrors to this SMI providing weak feedback light. Even only a fraction of reflected light enters into laser cavity, SMI system will maintains high accuracy. In addition, filters and algebraic operations save the computational expense for the purpose of real-time implementation.

Three conditions restrain this SMI: Response speed of used photoelectric detector, slewing rate of EOM and sampling rate of DAQ devices. Si-based PD in experiment response to impinged back-scattered light at nano-second rate(about $14 \mathrm{~ns}$ ). Since fluctuation of laser intensity by SMI effect is a steady state photonic-microwave signal, Doppler frequency shift of SMI is much smaller than response speed of PD. Slewing rate of EOM depends on applied voltages, which is upper to $0.25 \mathrm{GHz}$, the used slewing rate at $40 \mathrm{KHz}$ is sufficient since vibrating frequency of PZT is limited within $400 \mathrm{~Hz}$. Key restriction is sampling rate limited within $2 \mathrm{MHz} / \mathrm{s}$, based on Nyquist sampling theory, available maximum slewing rate is about $500 \mathrm{KHz}[18]$. It is notable that Doppler frequency of SMI is linearly proportional to measured velocity, so, measurable velocity range is within $2.5 \mathrm{~mm} / \mathrm{s}$. However, by using DAQ devices with higher sampling rate and sufficient slewing rate of EOM, even MHz vibration can be measured.

\section{Conclusion}

In presence, we introduced a carrier-separating method associated with EMDWT algorithm to simplify signal processing and improve SNR. Only algebraic operations and filters were necessary consuming much less time than harmonics analysis by FFT. In addition, strong dependence on electronic circuits was deprived due to signal processing operating in pure digital time domain, making this method very easy to duplicate. As demonstrated in experiment, carrier-separating technique was capable of distinguishing the vibration orientation automatically with non-cooperative objectives, which promise a high-efficiency and high-sensitivity signal processing. The operating principle announced the optimal phase modulation depth is 0.5rad. In addition, noise factors influencing measurement results had been analyzed and agreed with the experimental results. SNR degradation was suppressed by EMDWT which was summarized as: 1. The extracted quadrature signals were decomposed into stationary IMFs to reject noise components. 2. Recombined signals were decomposed by three level wavelet transform with Db series wavelet. Moreover, comparison with Polytec-5000 LDV demonstrated that this SMI was capable of measuring different shape vibrations dynamically with high resolution of nanometer scale. Signal process featured a repetitive measuring error of $1.5 \mathrm{~nm}$ which shown a potential value for industrial applications, especially in the dynamical measurement conditions.

\section{Funding Information}

National Natural Science Foundation of China (61178044); National Science Foundation of Jiangsu Province (BK20140925, BK2015...) and the University innovation project of Jiangsu Province (KYLX5_0724)

\section{Acknowledgment.}

We thank all reviewers for giving constructive instructions.

\section{References}

1 P. G. R. King and G. J. Steward, “Metrology with an optical maser,” New Sci. vol.17, pp.180-182,1963.

2 R. Lang and K. Kobayashi, "External optical feedback effects on semiconductor injection laser properties," IEEE J. Quan-tum Electron. Vol.QE-16, pp. 347-355, 1980. 
3 D. Lenstra, V. M. Van, and B. Jaskorzynska, "On the theory of a single-mode laser with weak optical feedback," Physica B \& C vol.125, pp. 255-264,1984.

4 P. J. D. Groot, G. M. Gallatin, and S. H. Macomber, "Ranging and velocimetry signal generation in a backscatter-modulated laser diode," Appl. Opt., vol. 27, pp.4475-4480, 1988.

5 R. W. Tkach and A. R. Chraplyvy, "Regimes of feedback effects in 1.5-m distributed feedback lasers," J. Lightwave Technol., vol. LT4, pp. 1655-1661,1986.

6 W. M. Wang, W. J. O. Boyle, K. T. V. Grattan, and A. W. Palmer, "Self-mixing interference in a diode laser: experimental observations and theoretical analysis," Appl. Opt., vol.32, pp. 1551- 1558,1993.

7 L.Scalise, Y.G. Yu, G. Giuliani, G. Plantier, T. Bosch, "Self-mixing laser diode velocimetry: application to vibration and velocity measurement, ,'IEEE T. Instrum. Meas. ,vol.53, pp.223-32, 2004.

8 D. Karabacak, T. Kouh and K.L. Ekinci. "Analysis of optical interferometric displacement detection in nanoelectro mechanical systems", .Appl.Phys., vol.9,1998.

9 C. Bes, V. Belloeil, Guy Plantier, Y. Gourinat and T. Bosch, "A Self-Mixing Laser Sensor Design with an Extended Kalman Filter for Optimal Online Structural Analysis and Damping Evaluation,” IEEE/Asme Transactions on mechatronics, vol.12, no.3, 2007.

10 M.L. Chen, Y. B. Zhang, C.X. Chen, L. Wang, W.C. Huang, "Damping Micro Vibration Measurement Using Laser Diode SelfMixing,” IEEE Photonics J., vol.6, no.4, 2014.

11 S. Donati, M. Norgia, “Self-Mixing Interferometry for Biomedical Signals Sensing,” IEEE J .Sel. Top. Quant., vol.20, no.2, 2014.

12 S. Shinohara, H. Yoshida, H. Ikeda, K. Nishide, and M. Sumi, "Compact and high-precision range finder with wide dynamic range and its application,” IEEE Trans Instrum. Meas. vol.41, pp.40-44, 1992.

13 H. Sun, J.G. Liu, Q. Zhang and R. Kennel, "Self-mixing interferometry for rotational speed measurement of servo drives," Appl. Opt.vol.55, pp.236-241,2016.

14 M. Nikolic, J. Dejan p, L. Yah Leng, B. Karl, T. Thomas, R. Aleksander D, "Approach to frequency estimation in self-mixing interferometry: multiple signal classification,” Appl. Opt., 2013, vol. 52, no 14, p. 3345-3350, 2013.

15 A. Magnani, D. Melchionni, A. Pesatori, M. Norgia, "Self-mixing digital closed-loop vibrometer for high accuracy vibration measurements,"Opt. Comm.,vol.365,pp.133-139, 2016.

16 N. Servagent, T. Bosch, and M. Lescure, "Design of a phase- shifting optical feedback interferometer using an electro optic modulator,” IEEE J. Sel. Top. Quantum Electron.vol. 6, pp.798-802,2000.

17 D. Guo, M. Wang, and S. Tan, "Self-mixing interferometer based on sinusoidal phase modulating technique," Opt. Express vol.13, no.5, pp.1537-1543,2005.

18 P. Groot, "Design of error-compensating algorithms for sinusoidal phase shifting interferometry,"Appl. Opt., vol.48, pp.67886796,2009 .

19 X. Wei, Q. Liu, H. Hao, D.M. Guo, M. Wang, X.Z. Chen, "Sinusoidal phase-modulating self-mixing interferometer with nanometer resolution and improved measurement velocity range," Appl. Opt., vol. 54, no. 26, 2015.

20 B. Ovryn and J. H. Andrews, “Phase-shifted laser feedback interferometry,” Opt. Lett., vol. 23, pp.1078-1080 ,1998.

21 B. Ovryn and J. H. Andrews, "Measurement of changes in optical path length and reflectivity with phase-shifting laser feedback interferometry,” Appl. Opt., vol.38, pp.1959-1967,1999. 
22 F. Azcona, R. Atashkhooei,S. Royo,J. Mendez Astudillo,A. Jha, “A nanometric displacement measurement system using differential optical feedback interferometry,” IEEE. Photonic. Tech. Lett., vol. 25, no 21, pp.2074-2077,2013.

23 L.David, G. Anders, H. Jorn M, B. Anja and Y. Kresten, "Self-mixing interferometry in vertical-cavity surface-emitting lasers for nanomechanical cantilever sensing,” Appl. Phys. Lett., vol.94, no.9, 091103,2009.

24 K. Kou,L. Xingfei, L. Li , "Injected current reshaping in distance measurement by laser self-mixing interferometry,” Appl. Opt., vol. 53, no.27, pp. 6280-6286,2014.

25 S. Donati, "Developing self-mixing interferometry for instrumentation and measurements," Laser Photon. Rev., vol. 6, pp. 393-417, 2012.

26 T. H. Peek, P.T. Bolwjin and C. Th. J. Alkemade , “Axial mode number of gas lasers from moving-mirror experiments,” Am. J. Phys. vol.35, pp.820-831, 1967.

27 G. Liu, S. Zhang and J. Zhu, "Theoretical and experimental study of intensity branch phenomena in self-mixing interference in a HeNe laser,” Opt. Commun. vol.221, pp.387-393, 2003.

28 S. Donati, "Laser interferometry by induced modulation of the cavity field," J. Appl. Phys., vol.49, pp.495-497,1978.

29 K. P. Birch and M. J. Downs, “An updated Edlén equation for the refractive index of air,” Metro.,vol. 30,pp. 155-162 ,1993.

30 JD Ellis, D Voigt, JW Spronck, AL Verlaan, RHM Schmidt, "Frequency stabilized HeNe gas laser with $3.5 \mathrm{~mW}$ from a single mode,"Prec. Eng., vol.36,no.2,pp.303-209,2012.

31 J. Lawall, J.M. Pedulla, Y.L. Coq, “Ultrastable laser array at $633 \mathrm{~nm}$ for real-time dimensional metrology,” Rev.Sci.Instrum.vol.72. no.7,pp.2879-2888, 2001.

32 S. Donati, G. Martini, T. Tambosso, "Speckle pattern errors in self-mixing interferometry," IEEE J. Quant. Electr., vol.39, pp.798 806, 2013.

33 M.Norgia, S. Donati, D. Alssandro, "Interferometric Measurements of Displacement on a Diffusing Target by a Speckle Tracking Technique,’IEEE J. Quant. Electr.,vol.37,no.6,pp.800-806,2001.

34 S. Donati, G. Martini, T. Tambosso, "Speckle pattern errors in self-mixing interferometry,” IEEE J. Quant. Electr., vol.39, pp.798 806, 2013.

35 S.Donati, G.Martini, "Systematic and random errors in self-mixing measurements: effect of the developing speckle statistics,"Appl. Opt.,vol.53,no.22,pp.4873-4880,2014.

36 Y.Ying, L. Xingfei, K. Ke "“Application of wavelet analysis in laser self-mixing interferometry for displacement measurement,” J. Optoelectron.Las., vol.6,pp.010, 2013.

37 S.Yuan, Y.Yanguang, X.Jiangtao,"Wavelet transform based de-noising method for self mixing interferometry signals," In : Asia Pacific Optical Sensors Conference. International Society for Optics and Photonics, pp. 83510G-83510G-7, 2012.

38 B.Olivier D, S. Han Cheng, Z.Usman, S.Frederic, "Robust fringe detection based on bi-wavelet transform for self-mixing displacement sensor," Sensors, IEEE, pp. 1-4,2015.

39 A. Jha,F.Azcona,C. Yañez,S. Royo, "Extraction of vibration parameters from optical feedback interferometry signals using wavelets," Applied Optics ,vol.54, no.34,2015.

40 N.E. Huang, Z. Shen, S.R. Long, M. Wu, H. Shih, N. Zheng, C. Yen, C.C. Tung, H.H. Liu, "The empirical mode decomposition and the Hilbert spectrum for non-linear and non-stationary time series analysis," Proc.R.Lond.A - Mathe. Phys. Eng. Sci. vol.454, pp903905,1998. 